\title{
Allogeneic Hematopoietic Progenitor Cell Transplantation - a Network of Medicine and a Chance for Transfusion Medicine
}

In this issue of Transfusion Medicine and Hemotherapy a 'Historical Perspective of Allogeneic Hematopoietic Cell Transplantation' is given by Rainer Storb, one of the pioneers of allogeneic hematopoietic progenitor cell transplantation (HPCT). He describes very clearly the astounding development during the past 50 years from the initial bone marrow transplantation with intensive radio- and chemotherapeutic conditioning regimens as a high-risk treatment approach for a very selected cohort of patients to a standardized peripheral blood stem cell transplantation with reduced conditioning as today's treatment of choice for an ever growing spectrum of patients. The time is long gone that HPCT was restricted to the treatment of young patients suffering from malignant diseases, and the shift from myeloablation to adoptive cellular immunotherapy continues.

In the beginning an intricate network of different medical specialties was mandatory to overcome the almost 'insurmountable complications' of allogeneic hematopoietic stem cell transplantation. In order to eradicate a potential residual tumor load and to allow for a stable engraftment of the bone marrow transplant, high-dose chemotherapy combined with total body irradiation was required. The resulting long-lasting myeloaplasia necessitated close interactions with the infectious disease department and the department of transfusion medicine for the complex supportive care with antiinfectious agents and various blood components in the often HLA-immunized patients. Over weeks of hospitalization extensive laboratory surveillance was necessary, sometimes followed by invasive procedures, organ biopsies, and immunohistochemical analysis to recognize beginning organ failures and graft-versushost disease (GvHD). Reverse isolation was used to protect the immunodeficient transplant recipients from opportunistic infections but was poorly tolerated by many a patient who required intensive psychological support to cope with the isolation in addition to the life-threatening disease and treatment.

In the early 1990s a new era began with the advent of G-CSF-mobilized peripheral blood hematopoietic progenitor cells (HPCs) as an alternative source for HPCT, which resulted in

- faster hematologic and immunologic reconstitution following transplantation,
- reduced risk for the recipient and shortened hospitalization,

- increased stem cell doses allowing for reduced conditioning regimens and better manipulation procedures of the graft.

These developments were paralleled by the improvement of HLA typing. New molecular techniques resulted in enhanced resolution of donor HLA characteristics, leading to a reduction in severe GvHD and transplant rejection. This laid the basis for a worldwide foundation of unrelated HPC donor registries. Due to improved registry networking, international logistics and the possibility of ambulatory peripheral blood HPC collection, more than $75 \%$ of allogeneic transplants performed in the last year in Germany were peripheral blood HPCs of which more than $50 \%$ originated from unrelated donors. The organization of registries by blood collection centers, the apheresis procedure required to collect peripheral blood HPCs, and changes in the legal status of these peripheral HPCs as a pharmaceutical product gave rise to an ever growing importance of the transfusion medicine specialist for the collection and preparation of peripheral blood stem cell transplants.

Rainer Storb's review further demonstrates the ongoing advance of HPCT, the development of methods as for the analysis of histocompatibility and chimerism, stem cell selection and expansion, transfection of blood cells as well as the generation of specific cellular immunotherapeutics which all are best allocated in the field of transfusion medicine. At present the primary focus lies on vaccination strategies based on cytotoxic $\mathrm{T}$ cells primed specifically against tumor or viral antigens, i.e. CMV and EBV, on NK cells in the haploidentical transplant setting, and on immune modulation using regulatory $\mathrm{T}$ cells or dendritic cells for the induction of graft-host tolerance. A well-known example is the transfusion of donor lymphocytes, which has become a standard procedure in nonmyeloablative HPCT.

Last but not least it is the plasticity of adult stem cells such as the HPCs and their ability to transdifferentiate that harbors many challenges and chances for today's transfusion medicine if we aim to make available engineered tissues or even organs to the clinician for the future.

M.M. Wiesneth, Ulm

\begin{tabular}{ll}
\hline KARGER & @ 2004 S. Karger GmbH, Freiburg \\
Fax+497614520714 & Accessible online at: \\
$\begin{array}{l}\text { E-mail Information@Karger.de } \\
\text { www.karger.com }\end{array}$ & www.karger.com/tmh
\end{tabular}

Dr. med. Markus M. Wiesneth

Institut für Klinische Transfusionsmedizin und Immungenetik

Universität Ulm

Helmholtzstraße 10, D-89081 Ulm

E-mail markus.wiesneth@medizin.uni-ulm.de 